\title{
Methodological limitations to a study on interpersonal therapy
}

To the Editor: Studies comparing pharmaco- v. psychotherapy in HIV-positive patients with depression are of great importance. These are vulnerable patients, both in a psychological sense and because of potential drug interactions. Psychotherapy should play an important role in the treatment of these patients.

A study by Moosa and Jeenah, ${ }^{[1]}$ published in the June 2012 issue of the South African Journal of Psychiatry, compared antidepressant treatment and interpersonal psychotherapy (IPT) in HIV-positive patients suffering from depression. A minimum of 5 and a mazimum of 12 sessions of IPT were administered. The mean Hamilton Depression Rating Scale (HAMD) scores of the pharmacotherapy group decreased from 25.7 to 6.2 and those of the psychotherapy group from 22.5 to 8.2.

We highlight, however, some methodological limitations to this study. The main limitation relates to the appropriate training for and administration of the IPT. The authors write that 'While the investigator was not trained or experienced in conducting IPT, it is our opinion that the therapy was correctly administered. ${ }^{[1]}$ The reporting of the correctness of the therapy merely as a matter of investigator opinion represents a weakness of the study.

IPT is a structured and manual-based psychotherapy usually consisting of 15 - 20 sessions over a 4 - 5-month period. ${ }^{[2,3]}$ Markowitz et al. ${ }^{[4]}$ did a study, published in the Archives of General Psychiatry, comparing IPT with other forms of therapy in HIV-positive patients suffering from depressive symptoms, in which patients were randomised to 16 weeks of treatment. They assured appropriate training and therapeutic rigor as follows: 'Experts in each modality trained therapists during a year-long phase, certifying them after at least 3 pilot cases. To avoid drift, therapists used treatment manuals and were monitored throughout with individual supervision of audiotaped sessions and blinded ratings of random sessions by independent, reliable adherence monitors. ${ }^{\text {[4] }}$

In the study by Markowitz et al., ${ }^{[4]}$ training was given over a 1 -year period. It appears that the therapist(s) described in the study described by Moosa and Jeenah ${ }^{[1]}$ did not receive training and had no experience in IPT.
Markowitz et al. ${ }^{[4]}$ note the problem of 'drift', i.e. practitioners straying from the principles and approach of a specific type of psychotherapy. The article by Moosa and Jeenah ${ }^{[1]}$ did not account for drift.

A further concern about Moosa and Jeenah's article is raised by the statements that 'cognitive behavioural therapy (CBT) can perform as well as antidepressants in treating patients with moderate to severe depression' (Introduction, paragraph 4) and that 'Our study also found IPT to be as effective as antidepressants in treating depression' (Discussion, paragraph 3). ${ }^{[1]}$ This may suggest mistakenly that even patients with severe depression could be treated adequately with CBT or IPT alone, without the use of an antidepressant.

Furthermore, the response to treatment in Moosa and Jeenah's ${ }^{[1]}$ study is high. The rigorous study by Markowitz et al. ${ }^{[4]}$ only demonstrated a remission rate of $59 \%$ for IPT. It is a limitation that Moosa and Jeenah do not comment on the overwhelmingly positive response to treatment in their study.

While it is important that South African data on the topic are published, readers should be mindful of this study's limitations in considering its clinical utility and in developing methodology for further research that aims to replicate these results.

\section{J L Roos}

\section{Krüger}

M W Böhmer

Department of Psychiatry, University of Pretoria, Gauteng, South Africa

1. Moosa MYH, Jeenah FY. Antidepressants versus interpersonal psychotherapy in treating depression in HIV-positive patients. South African Journal of Psychiatry 2012;18(2):47-52.

2. Sadock BJ, Sadock VI. Kaplan and Sadock's Comprehensive Textbook of Psychiatry. 8th ed. Philadelphia: Lippincott, Williams and Wilkins, 2005:2610-2619.

3. Markowitz JC. The clinical conduct of interpersonal psychotherapy. Focus 2006;4:179-184.

4. Markowitz JC, Kocsis JH, Fishman B, et al. Treatment of depressive symptoms in human immunodeficiency virus-positive patients. Arch Gen Psychiatry 1998;55(5):452-457.

S Afr J Psych 2013;19(2):55. DOI:10.7196/SAJP.457 